\section{An Efficient and Inexpensive System for Greenhouse Pot Rotation}

\author{
Erik M. Hardy ${ }^{1}$ and Dana M. Blumenthal \\ USDA Agricultural Research Service, Rangeland Resources Research Unit, \\ 1701 Center Avenue, Fort Collins, CO 80526
}

Additional index words. environmental variation, conveyor system, glass house

\begin{abstract}
Large greenhouse experiments require frequent pot rotation to minimize the effects of variation in greenhouse climatic conditions. A manual rotation process is often cumbersome and labor-intensive. To increase the efficiency of the rotation process, we propose an inexpensive, modular system fabricated from widely available equipment typically used in materials handling applications.
\end{abstract}

Large greenhouse experiments are often required to generate the necessary statistical power to adequately test scientific hypotheses. The results of these experiments can be confounded by variation in greenhouse light levels, temperature, and humidity, among other variables. For example, structural members, installed equipment, and tall vegetation adjacent to greenhouse walls can reduce solar radiation by up to $50 \%$ (Wang and Boulard, 2000) and produce variation sufficient to influence treatment responses (Guertal and Elkins, 1996). To address this issue, randomization and frequent rotation of pots within greenhouse experiments is necessary. Depending on the size of the experiment, however, a manual rotation process can be labor-intensive, cumbersome, and potentially damaging to the plants. Mechanized rotation systems have the potential to facilitate pot rotation (Kacira and Ling, 2001; Lazarovitch et al., 2006). The objective of this article is to describe an inexpensive, easily fabricated rotation system constructed from 24-inch $(61 \mathrm{~cm})$ skate wheel conveyor and wire pallet decking that can substantially reduce the time required for pot rotations. For example, in a recent experiment involving two greenhouse bays (three $1.4 \mathrm{~m}$ by $9.4-\mathrm{m}$ benches per bay) and 1200 pots, a full manual rotation took $10 \mathrm{~h}$ of labor. This time was reduced to 40 min using the skate wheel conveyor system described here.

\section{Design, Construction, and Use}

Our rotation system is comprised of a series of trays that rest on sections of skate wheel conveyor (Porta Power, Denver, CO). These trays are easily moved over the surface of the skate wheel conveyor, either individually or in rows of two to nine trays. The skate wheel conveyor is a common component of materials handling systems. It is used to move pallets, boxes, cartons, and other

Received for publication 14 Jan. 2008. Accepted for publication 20 Feb. 2008.

${ }^{1}$ To whom reprint requests should be addressed; e-mail Erik.Hardy@ars.usda.gov materials during the manufacturing and distribution process. The skate wheel conveyor comes in a variety of shapes and sizes, but the general design consists of a pair of structural side rails separated by crossmembers containing numerous skate wheels (Fig. 1). For our application, we used steel skate wheel conveyor sections that were $305 \mathrm{~cm}$ in length and $61 \mathrm{~cm}$ in width. Each $305-\mathrm{cm}$ section of steel skate wheel conveyor weighs $\approx 45 \mathrm{~kg}$, but this weight can be reduced by $30 \%$ to $40 \%$ if an aluminum skate wheel is used.

To use the skate wheel conveyor effectively, we had to fabricate trays to carry the pots used in the experiment. These trays were created using wire pallet decks (Porta Power), another standard, widely available component of materials handling systems, and pressure-treated $2 \times 6(5 \mathrm{~cm}$ by $15 \mathrm{~cm})$ lumber. Each tray consisted of a wire deck ( $94 \mathrm{~cm}$ by $124 \mathrm{~cm}$ ) with two pressure-treated wooden members installed perpendicular to the wire deck supports (Fig. 2). These wooden members provided two benefits: 1) the necessary surface area to allow for smooth movement of the trays over the skate wheel conveyor and 2) additional structural rigidity.

Figure 3 shows a rotation scheme using this system. To rotate, each tray is moved along the skate wheel conveyor the desired number of spaces. Because trays move freely over the skate wheel conveyor, even when heavily weighted, multiple trays can be moved simultaneously. For example, in our application, a tray plus 20 pots had a total weight of $\approx 59 \mathrm{~kg}$ and up to six trays could be moved at once. On reaching the end of one section, the tray is lifted from one bench to the next and begins traveling in the opposite direction. Designs in which rotation is conducted within sets of two benches (Fig. 3) are most efficient, requiring very little lifting. The system can also be adapted to an odd number of benches by using an S-shaped rotation. In this case, however, a subset of trays must be carried from the end to the beginning of the rotation. The number of pots per tray is constrained by the total weight of each tray and the spacing required between pots on a tray. Rotating trays between benches requires manually lifting trays from one bench to the next. Depending on tray weight with pots, pots can either be left in place or removed for this procedure.

Although frequent pot rotation should reduce the need for blocking, it can also be used in conjunction with blocking. For example, in large experiments, pairs of benches (Fig. 3) could comprise single blocks. If smaller-scale blocking is desired despite frequent rotation, blocks can be rotated together to minimize within-block variation.

One limitation of this system is that groups of pots move together on an individual tray. Thus, it does not minimize variation created by the pots themselves. Several steps can be taken to minimize this limitation. To deal with any aspect issues (created from greenhouse orientation to sun angle), trays can be periodically rotated $180^{\circ}$, easily done when switching benches. Given sufficient space, pot spacing can be increased to minimize effects of pots on neighboring pots. If these solutions are insufficient, pots can also be rotated within trays, or sizes of neighboring plants can be recorded and accounted for statistically using analysis of covariance.

The greatest benefit of this system is that pot rotation within a greenhouse is much faster and less labor-intensive. In our case, this system reduced the time needed for rotation by more than 10 -fold. This increase in efficiency increases the feasibility of 1) large greenhouse experiments and 2) frequent pot rotation. Another benefit of this rotation system is that it is versatile. The

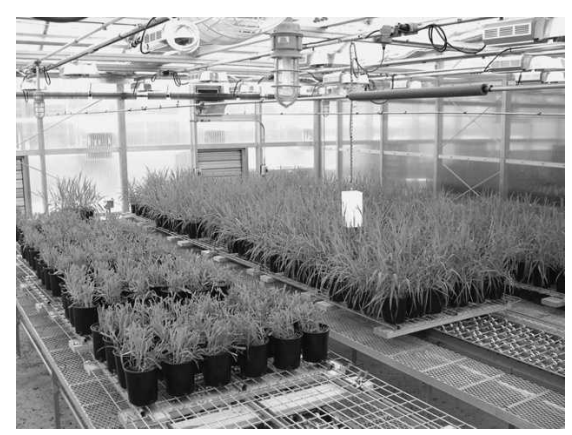

Fig. 1. The pot rotation system being used for invasive species research. The photograph shows the skate wheel conveyor, trays, greenhouse benches, and the overhead infrastructure, which can cause variation in greenhouse conditions.

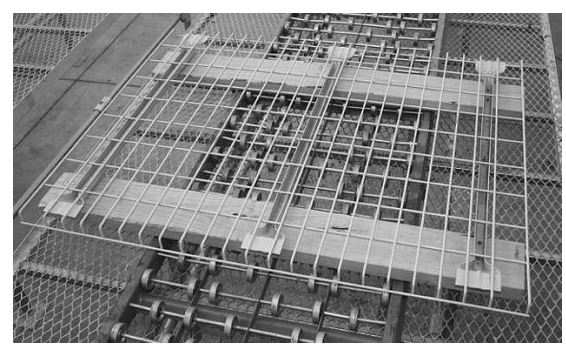

Fig. 2. A greenhouse rotation tray consisting of a wire deck $(94 \mathrm{~cm}$ by $124 \mathrm{~cm})$ with two pressure-treated wooden members installed perpendicular to the wire deck supports. 

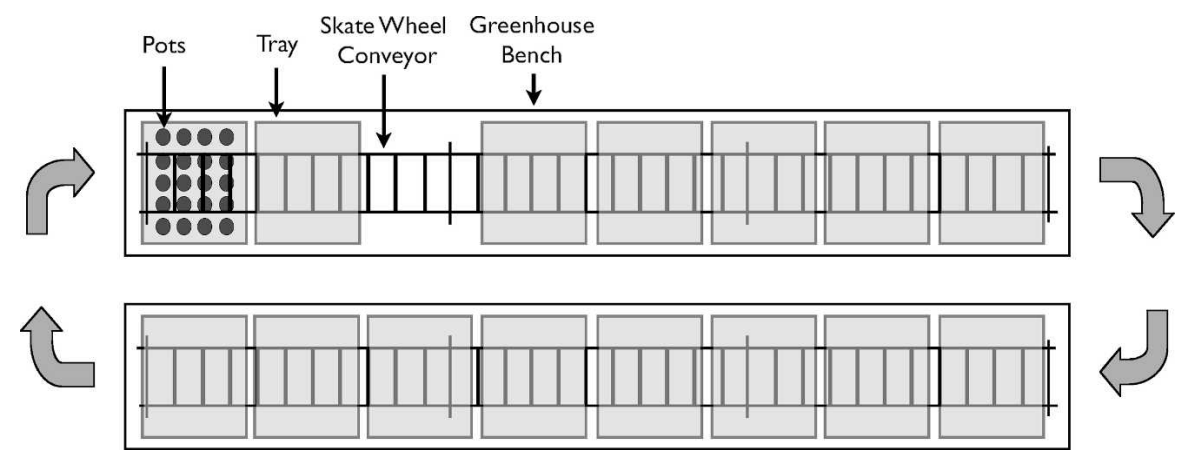

Fig. 3. A schematic representation of a greenhouse pot rotation system using two benches.

skate wheel conveyor can be placed on any horizontal surface, including the floor. It does not require any permanent installation, so the components of the system are easily moved between greenhouses. Also, the modular nature of the system allows it to be adapted to fit any size greenhouse and experiment. A third benefit of this rotation system is the low
$(61 \mathrm{~cm})$ skate wheel conveyor and 20 trays, was approximately $\$ 800$ U.S.. This one-time expense in materials is soon recouped by savings in the labor expense associated with the increased efficiency of the pot rotation process.

\section{Literature Cited}

Guertal, E.A. and C.B. Elkins. 1996. Spatial variability of photosynthetically active radiation in a greenhouse. J. Amer. Soc. Hort. Sci. 121:321-325.

Kacira, M. and P.P. Ling. 2001. Design and development of an automated and non-contact sensing system for continuous monitoring of plant health and growth. Trans. ASAE 44:989996.

cost. Because a majority of the components used in constructing the system are widely used products in large materials handling systems, both new and used materials are available from a variety of distributors. At the time of construction, materials cost for a system such as that shown in Figure 3, consisting of 18.2 linear meters of a 24 -inch

Lazarovitch, N., A. Ben-Gal, and S. Uri. 2006. An automated rotating lysimeter system for greenhouse evapotranspiration studies. Vadose Zone Journal 5:801-804.

Wang, S. and T. Boulard. 2000. Measurement and prediction of solar radiation distribution in full-scale greenhouse tunnels. Agronomie 20:41-50. 